\title{
Long-term monitoring of active stars.
}

\section{VI. $\boldsymbol{U} B \boldsymbol{V}(\boldsymbol{R I})_{\mathrm{c}}$ observations collected in Sep.-Oct. 1990 ${ }^{\star, \star \star}$}

\author{
G. Cutispoto ${ }^{1}$ and G. Leto ${ }^{2}$ \\ 1 Osservatorio Astrofisico di Catania, V.le A.Doria 6, I-95125 Catania, Italy \\ e-mail: gcutispoto@astrct.ct.astro.it \\ 2 Istituto di Rastronomia, C.P. 141, I-96017, Noto (SR), Italy
}

Received April 22; accepted June 6, 1996

\begin{abstract}
In the framework of an extensive program focusing on the global properties and evolution of active stars, high-precision $U B V(R I)_{\text {c }}$ photometry of 9 selected stars, collected at the European Southern Observatory over the intervals 7-17 September and 30 September - 10 October 1990, is presented. Significant evolution of the light curves, period variations and evidence for long-term variability of the global degree of spottedness are found. Some of the spectral classifications are discussed. These observations contribute to the establishment of a timeextended photometric database which can give important clues on topics such as the stability of the spotted areas, differential rotation, solar-like cycles and the correlation between inhomogeneities at different atmospheric levels.
\end{abstract}

Key words: stars: activity — stars: late-type — stars: variables

\section{Introduction}

The optical variability observed for the so-called active stars is generally accounted for by the presence of photospheric inhomogeneities, such as cool starspots, whose visibility, modulated by the stellar rotation, produces periodic or quasi-periodic light variation of the order of $0.1-0.2$ magnitudes in the $V$-band (cf. Rodonò 1986, 1992a,b and references therein). Multicolor photometry shows that color variations usually indicate a reddening of the star at minimum luminosity, thus supporting the cool starspot hypothesis. However, anticorrelation of the $U-B$ and $B-V$ color indices with respect to the

Send offprint requests to: G. Cutispoto

* based on data collected at the European Southern Observatory, La Silla, Chile.

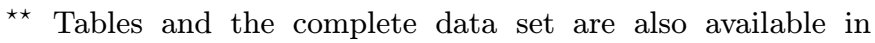
electronic form at the CDS via anonymous ftp to cdsarc.ustrasbg.fr or via http://cdsweb.u-strasbg.fr/Abstract.html
$V$-band light curve has been observed for some stars, i.e. V711 Tau, TW Lep and UX Ari (Cutispoto 1992; Rodonò \& Cutispoto 1992). The orbital or photometric periods of active stars span from less than one day to several weeks. The photometric waves can undergo noticeable changes over time scales as short as few stellar rotations (cf. Figs. 12, 18 and 23 in Cutispoto 1995); hence, in order to investigate the physical characteristics and evolution of spotted areas and the time scale of activity cycles, active stars must be observed systematically. This continuous monitoring program already started at Catania Astrophysical Observatory in the early Sixties and is carried out by using the 0.5 and $1.0 \mathrm{~m}$ telescopes of the European Southern Observatory (ESO, La Silla, Chile), the $0.25 \mathrm{~m}$ Automatic Photoelectric Telescope of Franklin \& Marshall College on Mt. Hopkins (AZ, U.S.A.) and the $0.8 \mathrm{~m}$ Automatic Photoelectric Telescope of Catania Astrophysical Observatory on Mt. Etna (Italy). It is aimed to establish the time-extended database that is essential to investigate fundamental topics such as the evolution of spotted areas and the correlation between inhomogeneities at different atmospheric levels (Rodonò et al. 1987; Pagano et al. 1992, 1993; Pallavicini et al. 1993; Küster et al. 1994). Long-term studies are also needed in order to search for the presence of photospheric solar-like activity cycles (see among others Cutispoto \& Rodonò 1992; Budding \& Zeilik 1995) and differential rotation (Rodonò 1992a). This paper reports on the results obtained by using the $0.5 \mathrm{~m}$ and the $1.0 \mathrm{~m}$ ESO telescopes and is organized as follows: details on the equipment and observations and reduction procedures are given in Sect. 2 , the results the and discussion on individual stars are presented in Sect. 3.

\section{The observations}

The present observations were carried out at the European Southern Observatory (La Silla, Chile) over the periods 


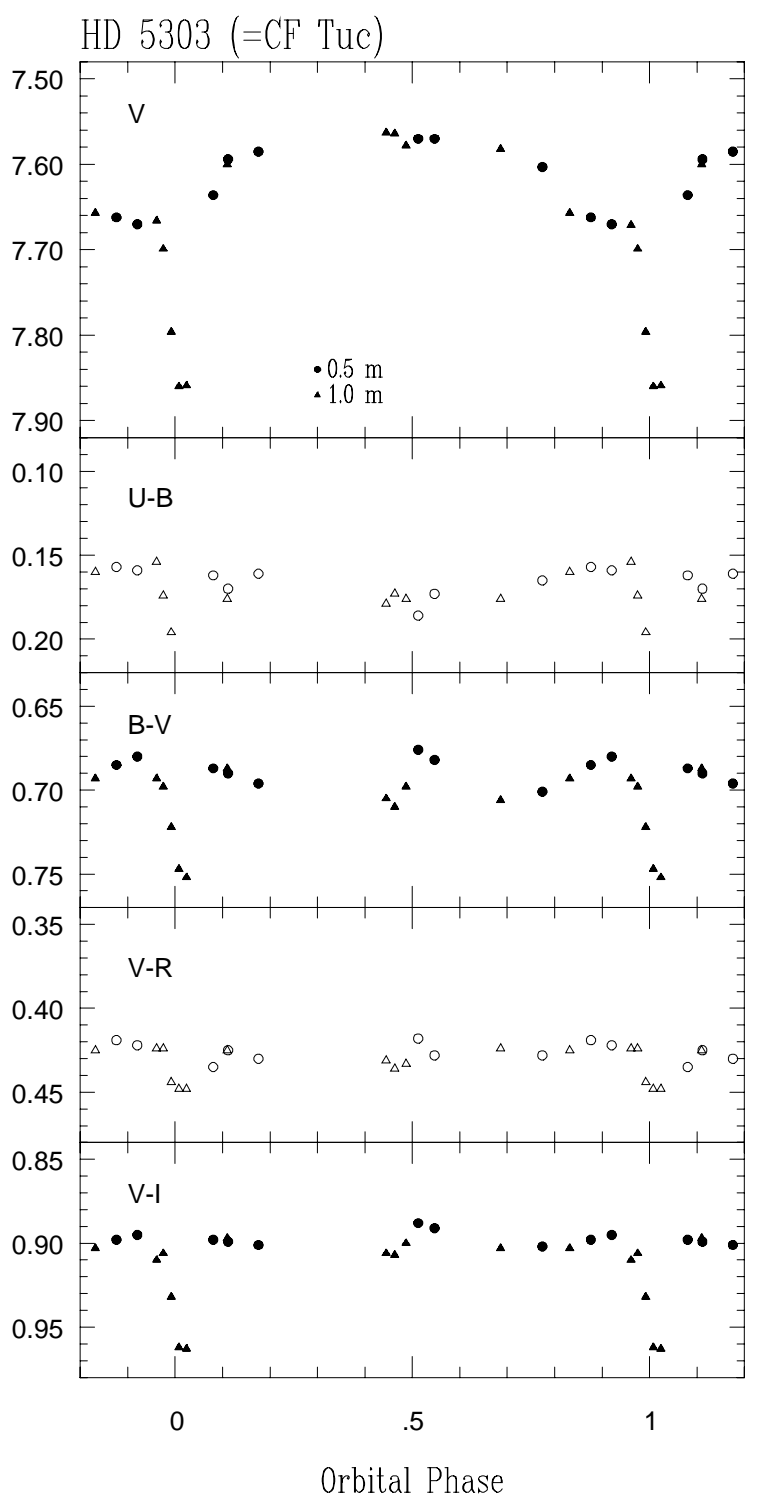

Fig. 1. $V$-band light curve and colors of $\mathrm{HD} 5303=\mathrm{CF}$ Tuc. Phases are reckoned from the spectroscopic ephemeris $\mathrm{HJD}=2445606.9165+2.797672 \cdot E($ Budding 1985)

Table 1. Comparison $(c)$ and check $(c k)$ stars used for each program star

\begin{tabular}{|ll|l|l|}
\hline \multicolumn{2}{|c|}{ Program Star $(v)$} & \multicolumn{1}{|c|}{$(c)$} & \multicolumn{1}{c|}{$(c k)$} \\
\hline HD 5303 & $=$ CF Tuc & HD 5210 & HD 6446 \\
HD 8435 & $=$ BC Phe & HD 8617 & HD 9896 \\
HD 16157 & $=$ CC Eri & HD 16371 & SAO 215945 \\
HD 32918 & $=$ YY Men & HD 33763 & HD 33747 \\
HD 155555 & $=$ V824 Ara & HD 156427 & HD 154775 \\
HD 174429 & $=$ PZ Tel & HD 173560 & HD 176557 \\
HD 197481 & $=$ AU Mic & HD 197237 & HD 197339 \\
HD 214479 & $=$ FK Aqr & HD 214046 & HD 214380 \\
HD 217344 & $=$ TZ PsA & HD 217108 & HD 217935 \\
\hline
\end{tabular}

7-17 September and 30 September - 10 October 1990, by using the $0.5 \mathrm{~m}$ and the $1.0 \mathrm{~m}$ ESO telescopes, respectively. Both instruments were equipped with a singlechannel photon-counting photometer, a thermoelectrically cooled Hamamatzu R-943/02 photomultiplier and standard ESO filters matching the $U B V(R I)_{\mathrm{c}}$ system. In order to obtain accurate differential photometry, for each program star $(v)$ a comparison $(c)$ and a check $(c k)$ star were also observed (see Table 1).

Each measurement consisted in the average of 10-15 1-sec. integrations in each filter, according to the color sequence $U-B-V-R-I$. A complete observation consisted

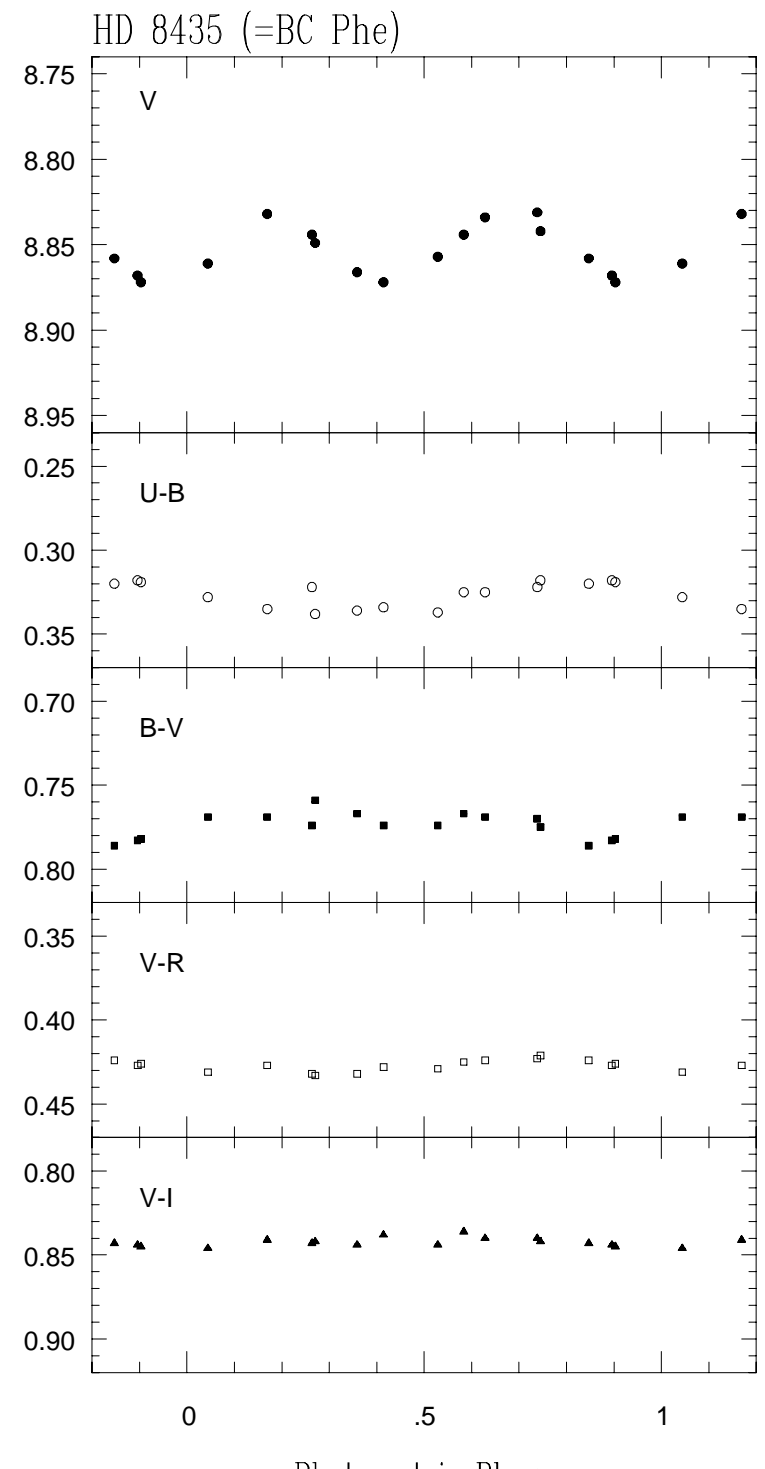

Photometric Phase

Fig. 2. $V$-band light curve and colors of $\mathrm{HD} 8435=\mathrm{BC}$ Phe. Phases are reckoned from the photometric ephemeris $\mathrm{HJD}=2447869.0+0.649 \cdot E($ Cutispoto 1995$)$ 
Table 2. Mean atmospheric extinction coefficients for La Silla site, obtained over the period 7 Sep. - 10 Oct. 1990

\begin{tabular}{|c|c|c|c|c|c|}
\hline & $U$ & $B$ & $V$ & $R$ & $I$ \\
\hline Mag/airmass & .460 & .217 & .125 & .087 & .041 \\
\hline
\end{tabular}

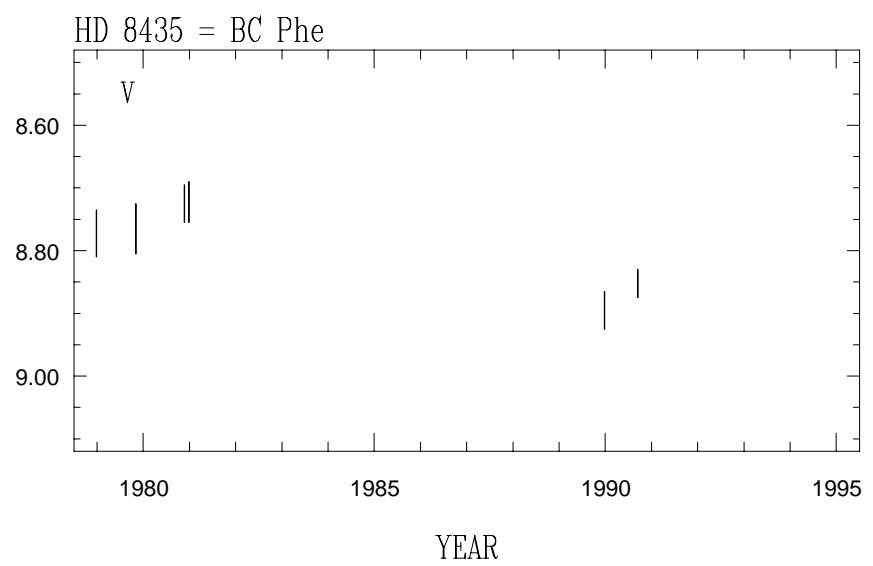

Fig. 3. $V$ light long-term variability of $\mathrm{HD} 8435=\mathrm{BC}$ Phe. The vertical bars indicate the peak-to-peak amplitude of the light curve

in sequential $c-v-v-v-v-c k-c$ measurements. From these data, four $v-c$ and one $c k-c$ differential magnitudes were computed; the four $v-c$ values were finally averaged to obtain one data point. The observations were corrected for atmospheric extinction and transformed into the standard $U B V(R I)_{\text {c }}$ system. The atmospheric extinction coefficients were obtained during clear nights by observing two standards of very different spectral type in the $1-2.5$ air mass range, while transformation coefficients were inferred by observing E-region standard stars (Menzies et al. 1989). The mean values of the extinction coefficients for La Silla are reported in Table 2. Note that these values have been obtained less than one year before the eruption of Mt. Pinatubo (June 1991). The typical error of our differential photometry is of the order of 0.005 magnitudes, with somewhat larger values (up to 0.01 magnitudes) in the $U$-band for the observations with the $0.5 \mathrm{~m}$ telescope due to the low photon counting level. The brighest $V$ magnitude and corresponding colors obtained for the program stars, and the standard deviations $(\sigma)$ for the $v-c$ and $c k-c$ mean differential $V$-band magnitudes obtained over $N$ nights are reported in Table 3 . The $V$ magnitudes and colors of the comparison and check stars were obtained via standard stars (Landolt 1983; Menzies et al. 1989; Menzies \& Laing 1988) and are given in Table 4. Taking into account the accuracy of the standard stars data and the extinction and transformation errors, the typical accuracy of the absolute photometry in Table 3 is of the order of 0.01 magnitude, with somewhat larger values (up to 0.02 magnitudes) for the $U-B$ color.

\section{Results}

The present multicolor photometry has been used to investigate the light curve evolutions with respect to previous observations, to search for the presence of photospheric solar-like activity cycles and, using the method described

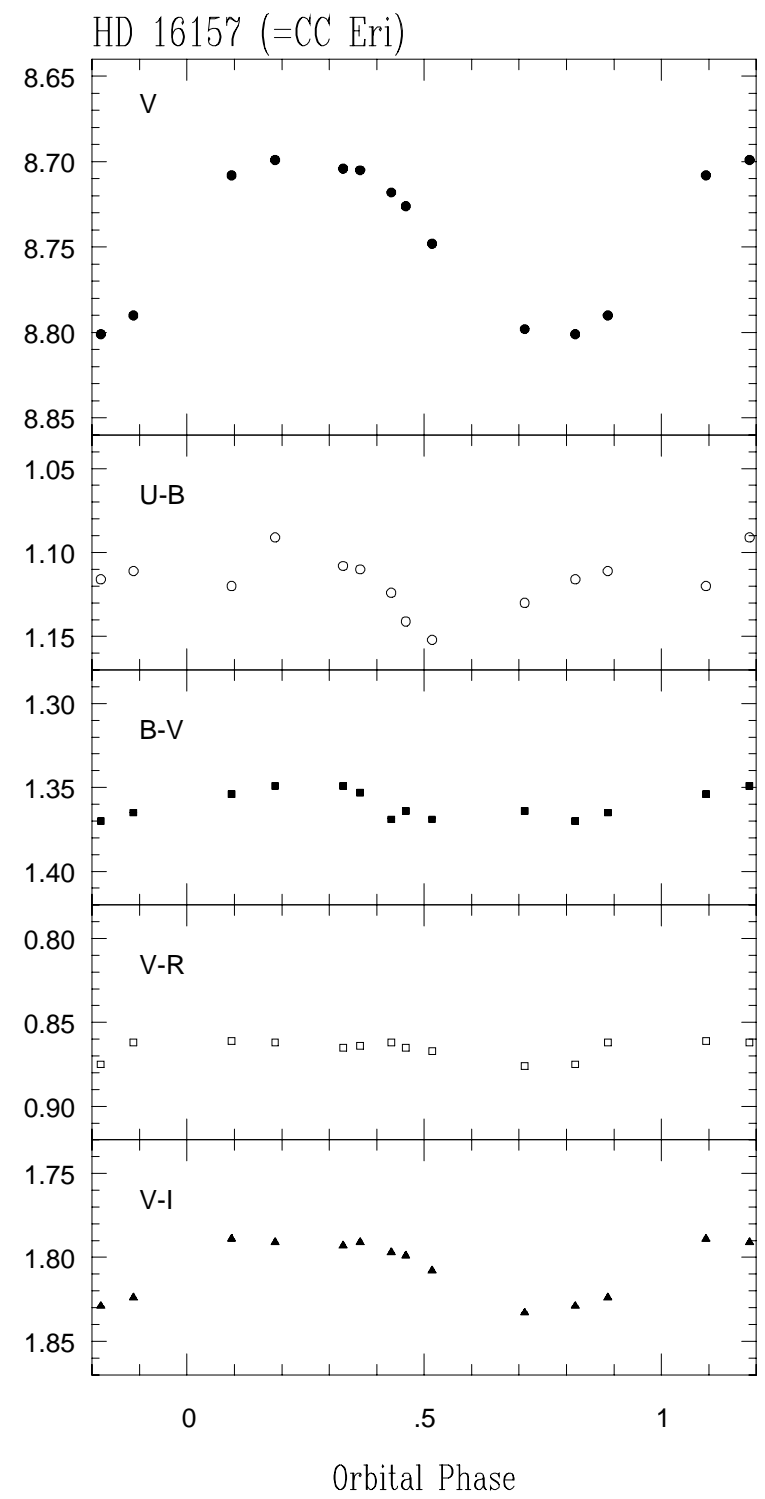

Fig. 4. $V$-band light curve and colors of HD 16157 $=\mathrm{CC}$ Eri. Phases are reckoned from the ephemeris HJD $=2447129.529+1.56145 \cdot E$, where the orbital period is from Evans (1959) 


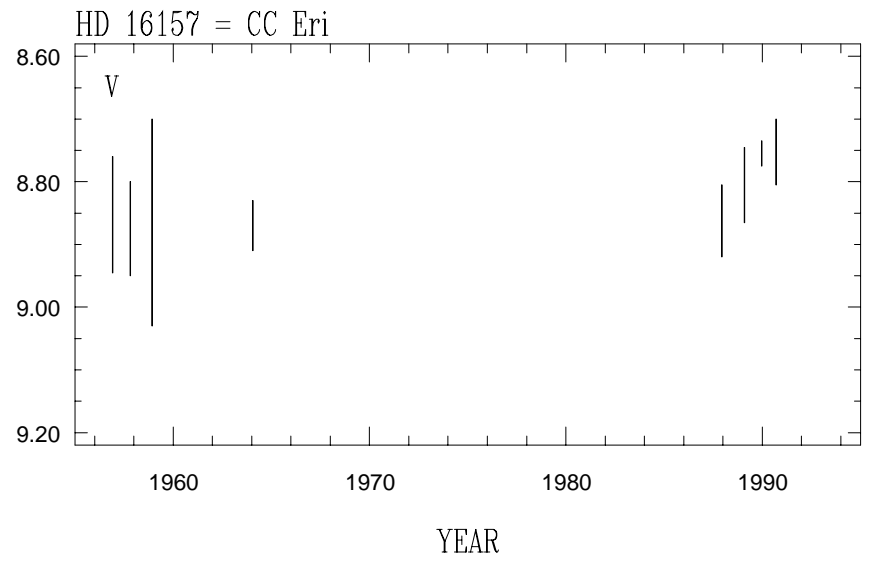

Fig. 5. $V$ light long-term variability of HD $16157=$ CC Eri. The vertical bars indicate the peak-to-peak amplitude of the light curve

by Cutispoto et al. (1996), to infer or further constrain the spectral type and luminosity class of the program stars (see Table 3). The results for the individual stars are discussed below.

HD $5303=\mathbf{C F}$ Tuc is a bright RS CVn-type eclipsing system that has been monitored by several authors in recent years (cf. Strassmeier et al. 1993; Cutispoto 1995; Budding \& Zeilik 1995 and references therein). It was detected during the ROSAT and EUVE all-sky EUV surveys (Pounds et al. 1993; Pye et al. 1995; Malina et al. 1994). The observations carried out with the $50 \mathrm{~cm}$ and the $1.0 \mathrm{~m}$ over the intervals 7-14 Sep. and 1-9 Oct., respectively are shown in Fig. 1, where phases are reckoned from the spectroscopic ephemeris HJD $=2445606.9165+2.797672 \cdot E$ (Budding 1985). The non-flat out-of-eclipse $V$-band light curve is indicative of the presence of photosperic spottedness and, with respect to previous observations (Cutispoto 1995; Budding \& Zeilik 1995 and references therein), displays a different luminosity maximum and shape. The remarkable light curve modifications observed for CF Tuc since late 1978 have been interpreted by Budding \& Zeilik (1995) in terms of a spot cycle lasting 5-6 years. The spectral classification of CF Tuc is not well established (see Cutispoto 1995 and references therein). The $v \sin i$ values reported by Randich et al. (1993) lead to stellar radii of about $1.46 R_{\odot}$ and $3.80 R_{\odot}$ for the hotter and cooler components, respectively while Coates et al. (1983) computed $1.69 R_{\odot}$ and $3.06 R_{\odot}$, respectively. These values indicate the presence of two evolved stars, both of luminosity class IV or V/IV. In fact, the best match of the observed colors is obtained by assuming the system to be formed by G0 V/IV + K4 V/IV or by G5 IV $+\mathrm{K} 4 \mathrm{~V} / \mathrm{IV}$ components, with the former solution in bet-

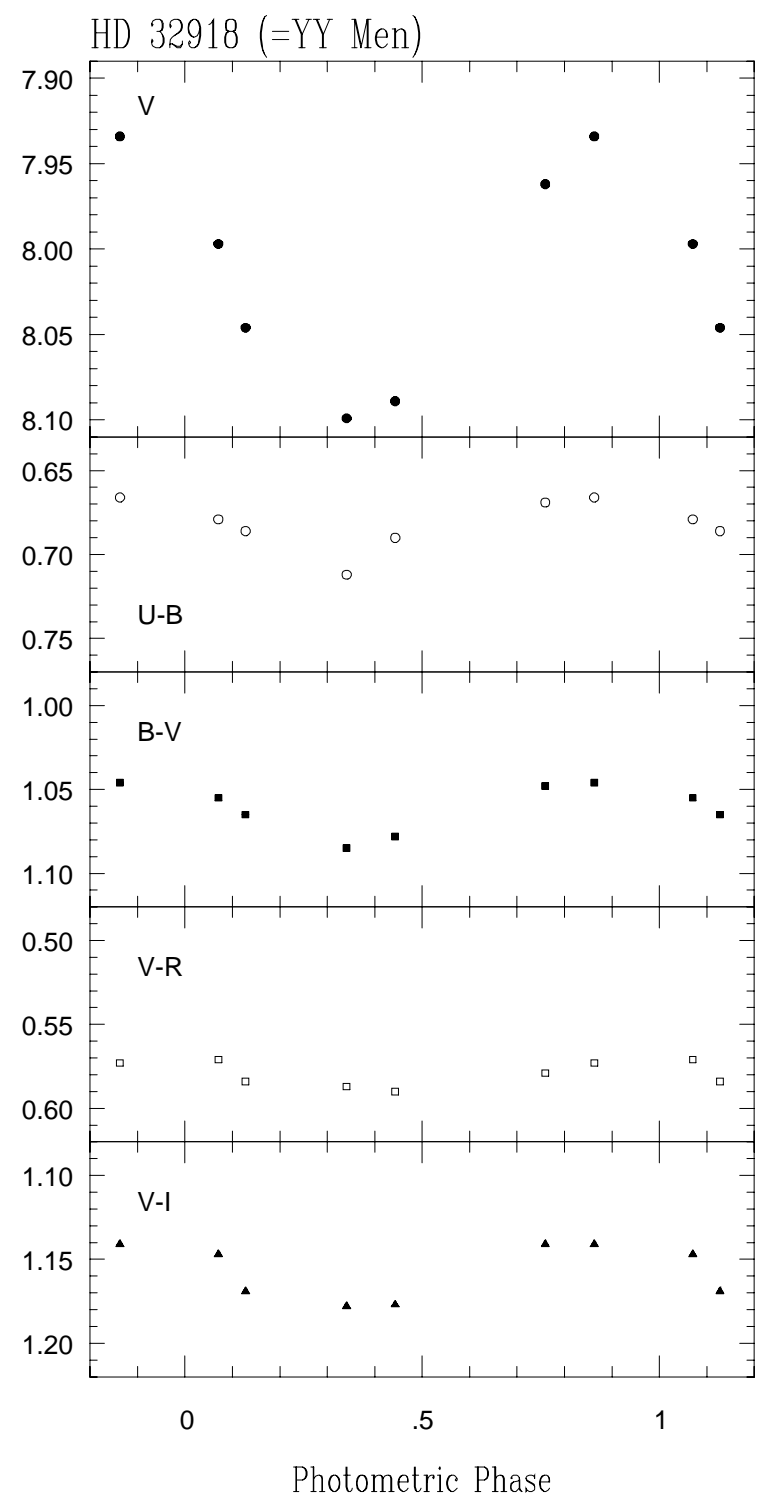

Fig. 6. $V$-band light curve and colors of HD $32918=\mathrm{YY}$ Men. Phases are reckoned from the photometric ephemeris $\mathrm{HJD}=2444155.64+9.5476 \cdot E($ Collier 1982)

ter agreement with the computed radii.

HD $8435=$ BC Phe is a SB2 spectroscopic binary with an orbital period of 0.657 days (Balona 1987). Photometric observations have been obtained by Lloyd Evans \& Koen (1987); Collier Cameron (1987) and Cutispoto (1995). Low Li abundance is reported by Pallavicini et al. (1992). No microwave emission was detected by Slee et al. (1987). The observations obtained with the $0.5 \mathrm{~m}$ over the interval 7-14 Sep. are shown in Fig. 2. The light curve has been folded by using the 0.649-day photometric period inferred by Cutispoto (1995). Comparing the data in Fig. 2 with those obtained about 9 months earlier by Cutispoto (1995), a remarkable evolution of the light curve is 
Table 3. Maximum luminosity $\left(V_{\max }\right)$ and corresponding colors for the program stars; standard deviations $(\sigma)$ for the $v-c$ and $c k-c V$-band differential magnitudes for each series of $N$ nights; inferred spectral classification (Spectral Type)

\begin{tabular}{|l|c|c|c|c|c|c|c|c|l|}
\hline $\begin{array}{c}\text { Program } \\
\text { Star }\end{array}$ & $V_{\max }$ & $U-B$ & $B-V$ & $V-R_{\mathrm{c}}$ & $V-I_{\mathrm{c}}$ & $\begin{array}{c}\sigma \\
(v-c)\end{array}$ & $\begin{array}{c}\sigma \\
(c k-c)\end{array}$ & $\begin{array}{c}N \\
\text { Spectral } \\
\text { Type }\end{array}$ \\
\hline HD 5303 & 7.56 & 0.17 & 0.68 & 0.43 & 0.89 & 95 & 6 & 14 & $\mathrm{G} 0 \mathrm{~V} / \mathrm{IV}+\mathrm{K} 4 \mathrm{~V} / \mathrm{IV}$ \\
HD 8435 & 8.83 & 0.32 & 0.77 & 0.42 & 0.89 & 14 & 6 & 8 & $\mathrm{G} 7 / 8 \mathrm{~V} / \mathrm{IV}+\mathrm{K} 3 / 5 \mathrm{~V}$ \\
HD 16157 & 8.70 & 1.09 & 1.35 & 0.86 & 1.79 & 39 & 4 & 6 & $\mathrm{~K} 7 \mathrm{~V}+\mathrm{M} 1 \mathrm{~V}$ \\
HD 32918 & 7.93 & 0.67 & 1.05 & 0.57 & 1.14 & 62 & 3 & 6 & $\mathrm{~K} 1 \mathrm{III}$ \\
HD 155555 & 6.85 & 0.39 & 0.81 & 0.47 & 0.92 & 37 & 6 & 9 & $\mathrm{G} 5 \mathrm{IV}+\mathrm{K} 0 \mathrm{IV}$ \\
HD 174429 & 8.44 & 0.30 & 0.79 & 0.45 & 0.87 & 11 & 6 & 12 & $\mathrm{~K} 0(\mathrm{PMS})$ \\
HD 197481 & 8.70 & 1.13 & 1.47 & 0.94 & 2.06 & 25 & 10 & 7 & $\mathrm{M} 1 \mathrm{Ve}$ \\
HD 214479 & 9.09 & 1.08 & 1.46 & 0.94 & 2.19 & 16 & 5 & 12 & $\mathrm{M} 2 \mathrm{Ve}$ \\
HD 217344 & 8.46 & 0.20 & 0.71 & 0.41 & 0.81 & 11 & 3 & 8 & $\mathrm{G} 5 / 6 \mathrm{IV}+?$ \\
\hline
\end{tabular}

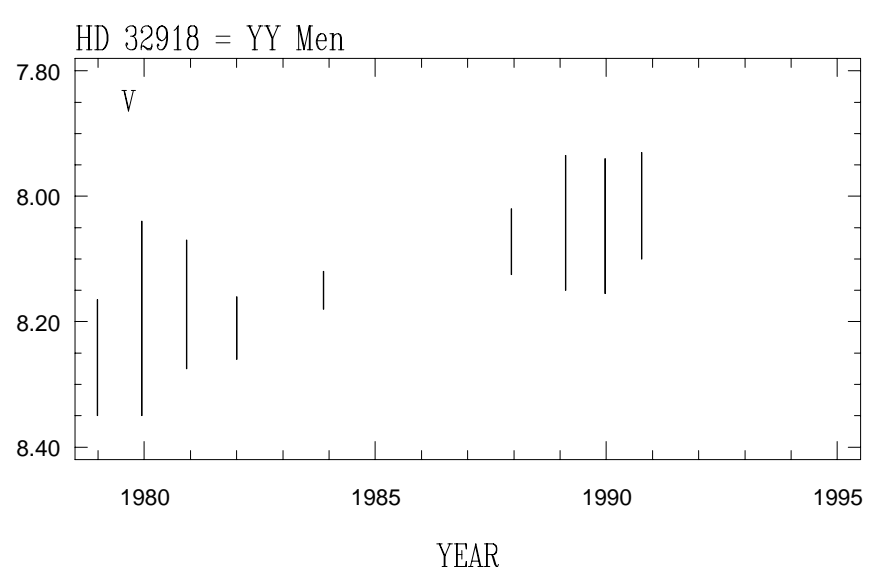

Fig. 7. $V$ light long-term variability of HD $32918=$ YY Men. The vertical bars indicate the peak-to-peak amplitude of the light curve

evident. In fact, the present data show two clear and almost identical maxima, while the global degree of spottedness has decreased, as can be inferred from the collection of the available photometry presented in Fig. 3. The colors are consistent with the $\mathrm{G} 7 / 8 \mathrm{~V} / \mathrm{IV}+\mathrm{K} 3 / 5 \mathrm{~V}$ spectral classification suggested by Cutispoto (1995).

HD $16157=$ CC Eri is a BY Dra-type variable that has been studied at different wavelengths by several authors (see Byrne et al. 1992; Cutispoto 1991 and references therein). More recently, it was detected by the ROSAT and EUVE satellites during their all-sky surveys (Pye et al. 1995; Malina et al. 1994; Pounds et al. 1993). The observations obtained with the $0.5 \mathrm{~m}$ over the interval 7-14 Sep. are shown in Fig. 4, where phases are reckoned from the 1.56145-day orbital period (Evans 1959). Clear color variations, partially correlated with the $V$-band modulation, are present for all the color indices. Comparing the present data with recent photometric observations (Cutispoto $1991 ; 1992 ; 1995)$ a remarkable decrease in the maximum luminosity emerges (cf. Fig. 5). In fact,

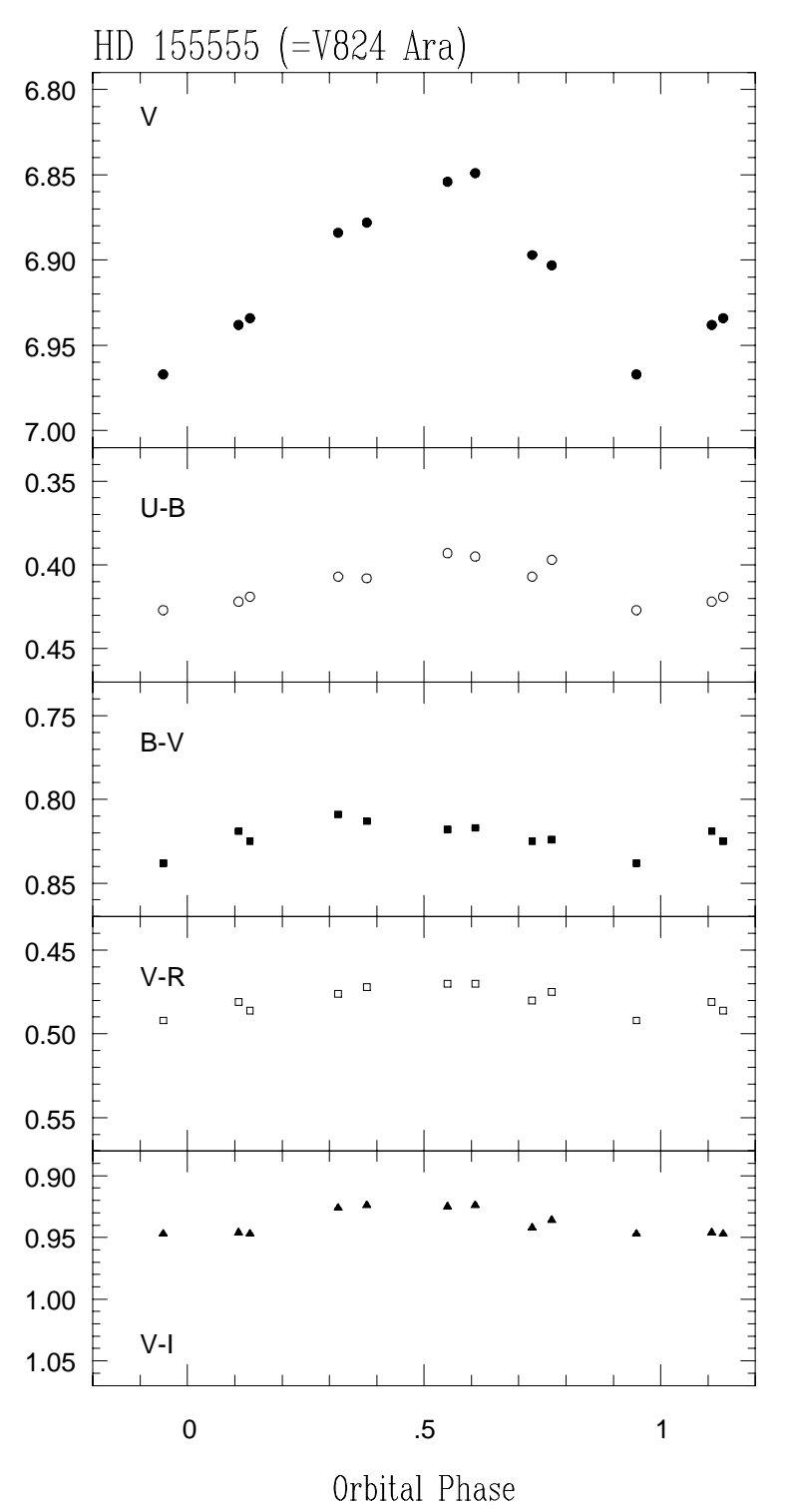

Fig. 8. $V$-band light curve and colors of HD $155555=\mathrm{V} 824$ Ara. Phases are reckoned from the spectroscopic ephemeris $\mathrm{HJD}=2445803.07+1.681652 \cdot E$ (Pasquini et al. 1991) 


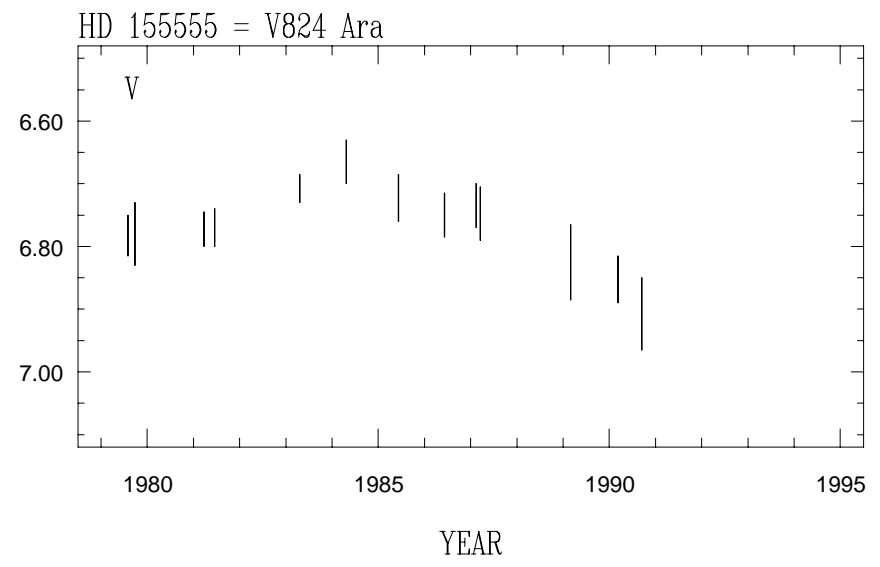

Fig. 9. $V$ light long-term variability of HD $155555=\mathrm{V} 824$ Ara. The vertical bars indicate the peak-to-peak amplitude of the light curve

the star has reached the same maximum luminosity observed in late 1958 (Evans 1959) that could be close to the unspotted $V$ magnitude value. The observed colors can be well matched by assuming a $\mathrm{K} 7 \mathrm{~V}+\mathrm{M} 1 \mathrm{~V}$ spectral classification.

Table 4. $V$ magnitude and colors for the $c$ and $c k$ stars derived via standard stars. Errors are of the order of 0.01 magnitudes. The symbol ":" denotes errors of the order of 0.02 magnitudes

\begin{tabular}{|l|l|l|c|c|c|}
\hline \multicolumn{1}{|c|}{$c$ or $c k$} & $V$ & $U-B$ & $B-V$ & $V-R_{\mathrm{c}}$ & $V-I_{\mathrm{c}}$ \\
\hline HD 5210 & 8.70 & $0.08:$ & 0.60 & 0.32 & 0.66 \\
HD 6446 & 7.24 & $1.37:$ & 1.38 & 0.72 & 1.44 \\
HD 8617 & 8.98 & $1.02:$ & 1.12 & 0.57 & 1.11 \\
HD 9896 & 6.00 & $-.02:$ & 0.38 & 0.21 & 0.44 \\
HD 16371 & 8.09 & $0.57:$ & 0.89 & 0.47 & 0.92 \\
SAO 215945 & 9.74 & $-.01:$ & 0.37 & 0.21 & 0.43 \\
HD 33747 & 8.75 & $0.74:$ & 1.00 & 0.52 & 1.02 \\
HD 33763 & 8.38 & $0.50:$ & 0.90 & 0.48 & 0.95 \\
HD 154775 & 7.60 & $1.97:$ & 1.59 & 0.86 & 1.75 \\
HD 156427 & 7.41 & $1.71:$ & 1.49 & 0.80 & 1.55 \\
HD 173560 & 8.71 & $0.11:$ & 0.64 & 0.36 & 0.70 \\
HD 176557 & 7.20 & $1.77:$ & 1.48 & 0.78 & 1.48 \\
HD 197237 & 9.00 & $0.84:$ & 0.99 & 0.50 & 0.96 \\
HD 197339 & 7.39 & $1.44:$ & 1.30 & 0.66 & 1.24 \\
HD 214046 & 7.54 & 1.78 & 1.43 & 0.76 & 1.48 \\
HD 214380 & 9.21 & -.02 & 0.48 & 0.30 & 0.57 \\
HD 217108 & 9.17 & $0.03:$ & 0.53 & 0.29 & 0.58 \\
HD 217935 & 8.59 & $0.72:$ & 0.97 & 0.50 & 0.96 \\
\hline
\end{tabular}

HD $32918=$ YY Men is an active giant with the typical characteristics of FK Com stars (see Cutispoto et al. 1992 and references therein). It has shown two of the most intense and longest duration radio flares (Slee et al. 1987; Bunton et al. 1989) and probably the longest

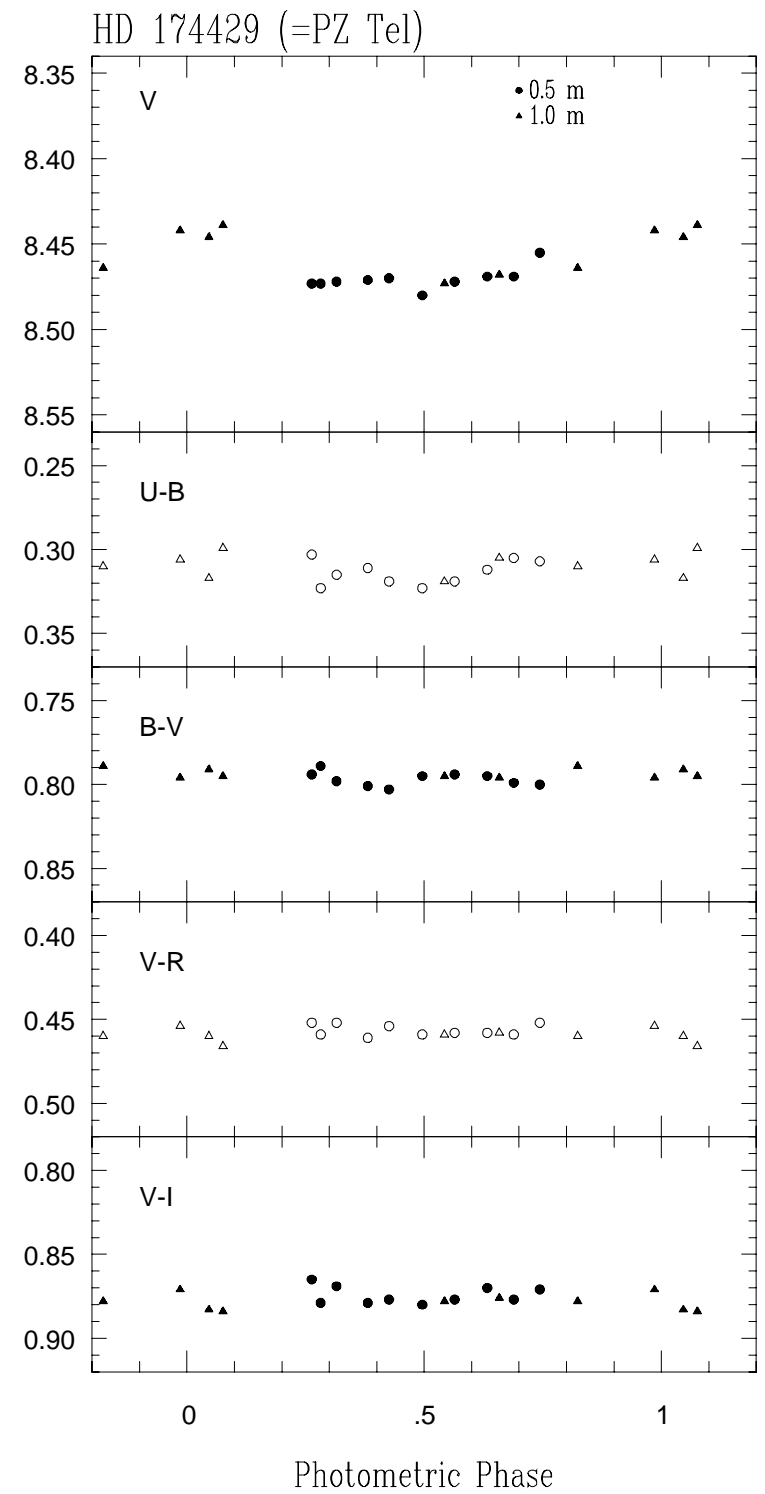

Fig. 10. $V$-band light curve and colors of $\mathrm{HD} 174429=\mathrm{PZ}$ Tel. Phases are reckoned from the ephemeris HJD $=2448141.5$ $+0.94486 \cdot E$, where the photometric period is from Innis et al. (1990)

optical flare ever recorded for any class of active stars (Cutispoto et al. 1992). The data obtained over the interval 1-10 Oct. by using the $1.0 \mathrm{~m}$ telescope are shown in Fig. 6. Phases are computed using the 9.5476 day photometric period inferred by Collier (1982). The $V$-band light curve is single-peaked and its maximum is probably close to the stellar unspotted level (cf Fig. 7). Remarkable color variations well correlated with the variability in the $V$-band are present and show the star to be redder at light minimum.

HD $155555=$ V824 Ara is a rapidly rotating SB2 spectroscopic binary that has been classified as a pre-main 


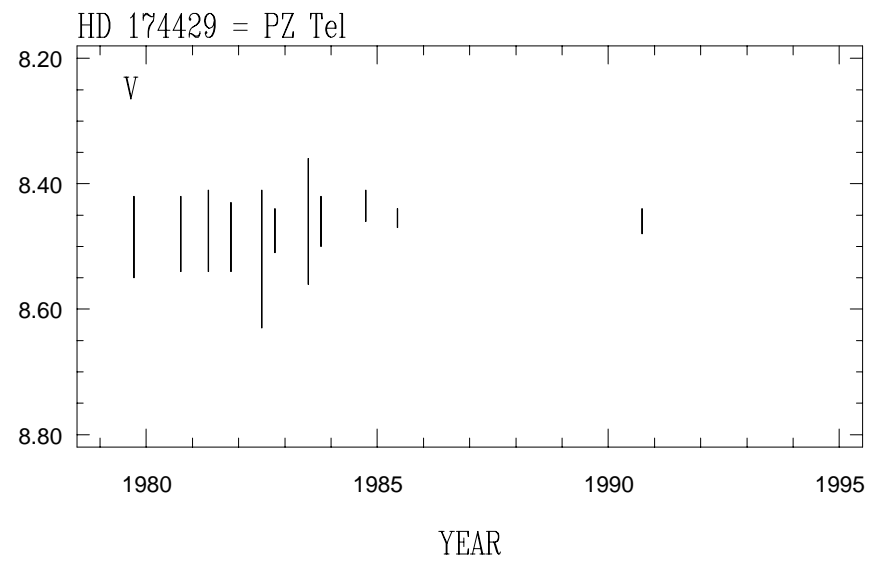

Fig. 11. $V$ light long-term variability of HD $174429=\mathrm{PZ}$ Tel. The vertical bars indicate the peak-to-peak amplitude of the light curve

sequence object by Pasquini et al. (1991) and by Martin \& Brandner (1995). It was detected by the ROSAT (Pounds et al. 1993; Pye et al. 1995) and EUVE (Malina et al. 1994) satellites during their all-sky EUV surveys and has been observed photometrically since late 1979 by several authors (see Cutispoto 1996 and references therein). The observations presented in Fig. 8 were obtained with the $0.5 \mathrm{~m}$ telescope over the interval 7-17 Sep. Any light contribution from LDS587 B, that lies 33 arcsec apart, was avoided. Phases have been reckoned from the 1.681652day orbital period computed by Pasquini et al. (1991). The light curve is single-peaked and has a different shape and a larger amplitude with respect to the light curve obtained less than seven months before (Cutispoto 1996). The colors, whose variations are in phase with the $V$-band modulation and show the star to be redder at minimum luminosity, are consistent with the G5 IV + K0 IV spectral classification reported by Pasquini et al. (1991). Finally, from the collection of the available photometry of V824 Ara shown in Fig. 9, we note that the present observations show the faintest $V$ brightness ever observed, confirming the presence of strong variations of the global degree of photospheric spottedness with a tentative period of at least 13 years.

HD $174429=\mathrm{PZ}$ Tel is a rapidly rotating late-type chromospherically active star that has been classified as a very young or as a pre-main sequence star (Innis et al. 1986). It shows a filled in $\mathrm{H} \alpha$ line (Innis et al. 1988) and Ca II H\&K emission lines (Bidelman \& MacConnell 1973; Houk 1978; Innis et al. 1988). The radial velocity data suggest that the star is single (Balona 1987; Innis et al. 1988). HD 174429 has been detected during a microwave survey of southern active stars (Slee et al. 1987) and shows a very high $\mathrm{Li}$ abundance (Randich et al. 1993). The optical variability was discovered by Coates et al. (1980), further photometric data can be found in Bopp et al. (1986) and

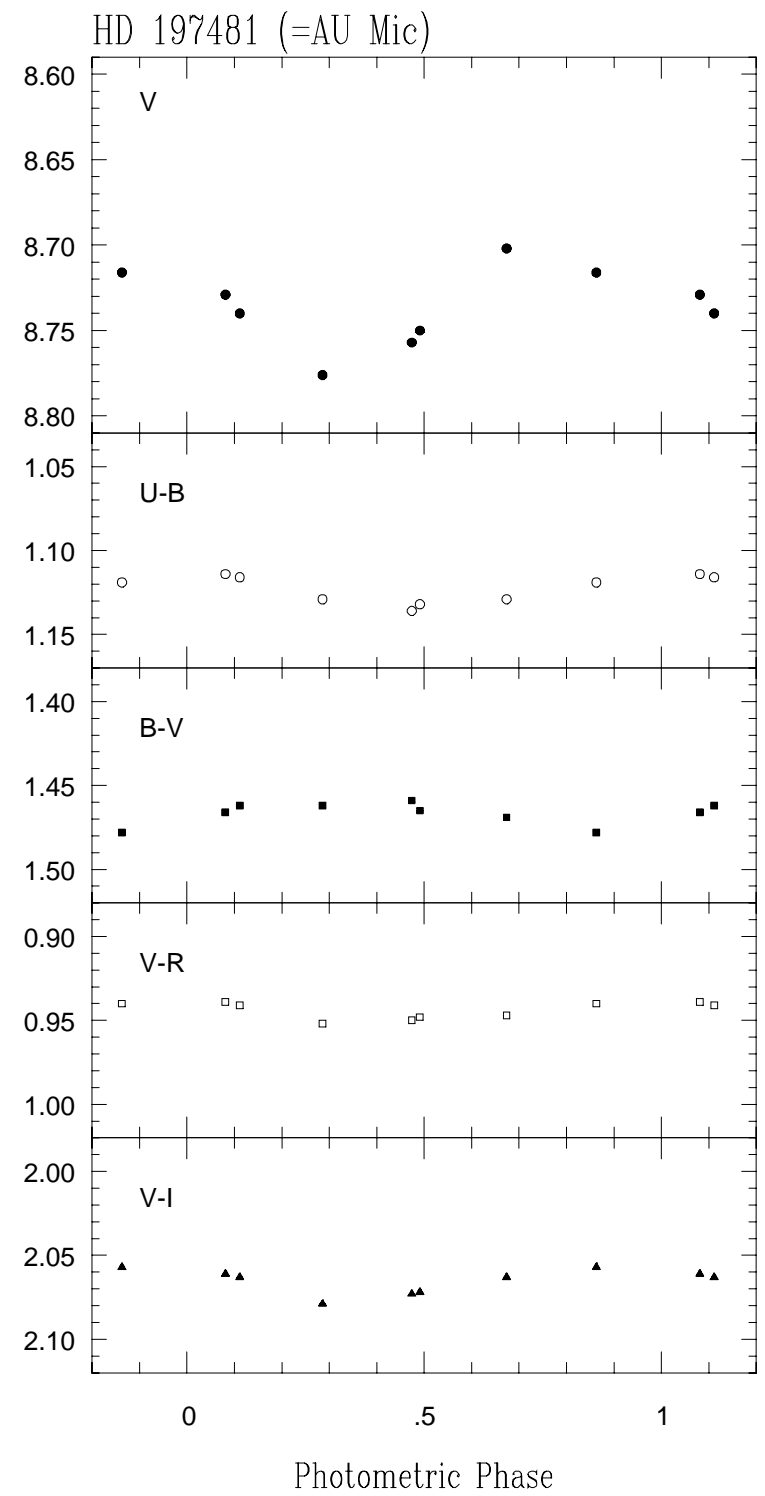

Fig. 12. $V$-band light curve and colors of HD $197481=\mathrm{AU}$ Mic. Phases are reckoned from the photometric ephemeris HJD $=2441051.0+4.865 \cdot E($ Torres et al. 1972)

Innis et al. (1990). The observations presented in Fig. 10 were obtained over the intervals 7-17 Sep. and 30 Sep. 10 Oct. with the $0.5 \mathrm{~m}$ and the $1.0 \mathrm{~m}$, respectively; phases have been computed by using the photometric period of 0.94486-day inferred by Innis et al. (1990). The light curve has a very low amplitude and no significant color variations are present. A collection of the available photometry of HD 174429 is shown in Fig. 11. From these data it is evident that the mean $V$ magnitude has been almost constant over the last 12 years. Houk (1978) classified PZ Tel as a K0 Vp star and our colors are in agreement with such a classification, with the noticeable exception of the $U-B$ color that is about 0.15 magnitudes bluer than expected. However, Randich et al. (1993) computed for the 


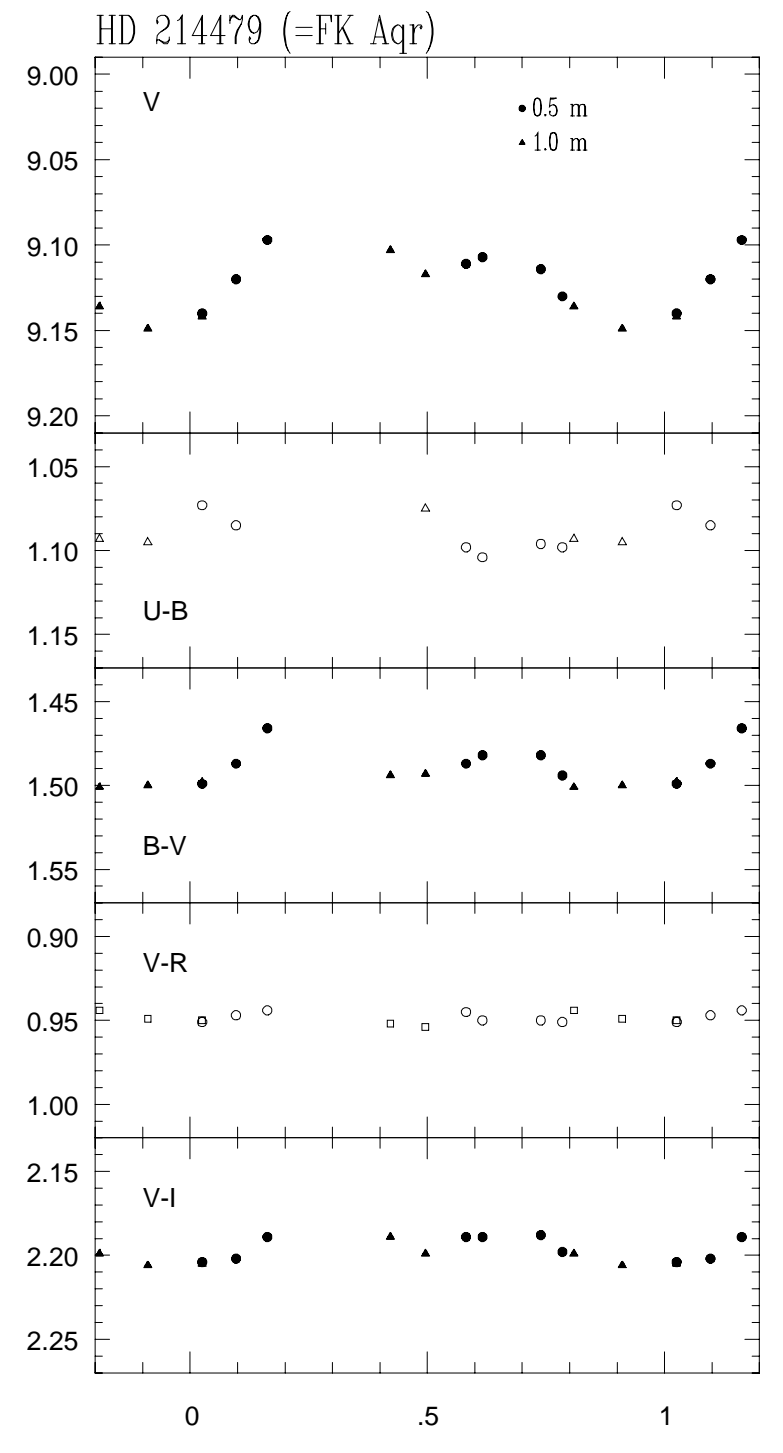

Photometric Phase

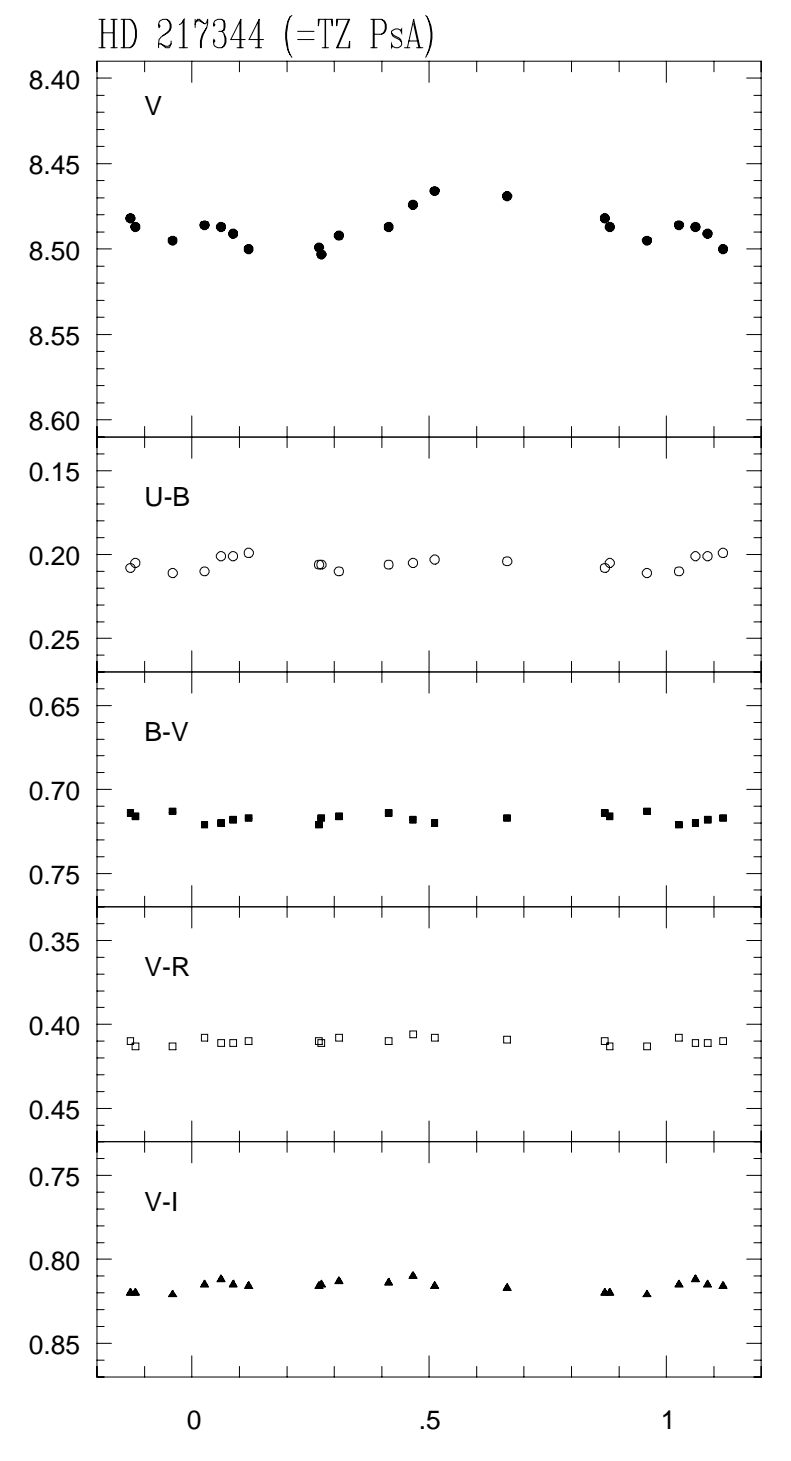

Photometric Phase
Fig. 13. $V$-band light curve and colors of HD $214479=\mathrm{FK}$ Aqr. Phases are reckoned from the photometric ephemeris $\mathrm{HJD}=2448141.0+4.252 \cdot E$

$v \sin i$ a value of the order of $70 \mathrm{~km} \mathrm{~s}^{-1}$, which leads to infer a minimum stellar radius of about $1.31 R_{\odot}$. This radius is too high for a luminosity class $\mathrm{V}$ star, confirming that HD 174429 is probably still approaching the main sequence.

HD $197481=$ AU Mic is a flare star that is also a member of the BY Dra-type variables. It shows strong emission lines indicative of the presence of an active chromosphere and transition region (Linsky et al. 1982; Quin et al. 1993; Houdebine \& Doyle 1994 and references therein). X-ray and EUV emission has been revealed by the Einstein (Golub 1983), ROSAT (Pounds et al. 1993; Pye et al. 1995) and EUVE (Malina et al. 1994) satellites. The
Fig. 14. $V$-band light curve and colors of HD 217344 $=\mathrm{TZ}$ PsA. Phases are reckoned from the ephemeris HJD $=2448141.54+1.648 \cdot E$, where the photometric period is from Lloyd Evans \& Koen (1987)

optical variability was discovered by Torres et al. (1972). The observations presented here were obtained using the $0.5 \mathrm{~m}$ telescope over the interval 7-17 Sep. They are shown in Fig. 12, where phases are reckoned from the photometric ephemeris HJD $=2441051.0+4.865 \cdot E$ (Torres et al. 1972 ). The colors, that show weak variations, are consistent with the M1 Ve spectral classification reported by Houk (1982). In particular, the $V-R$ and $V-I$ modulation is clearly in phase with the $V$-band variability.

HD $214479=$ FK Aqr is the brightest component of the visual pair ADS 11854 (= GL $867 \mathrm{AB}$ ) and is itself a spectroscopic SB2 binary with an orbital period of 4.08322 


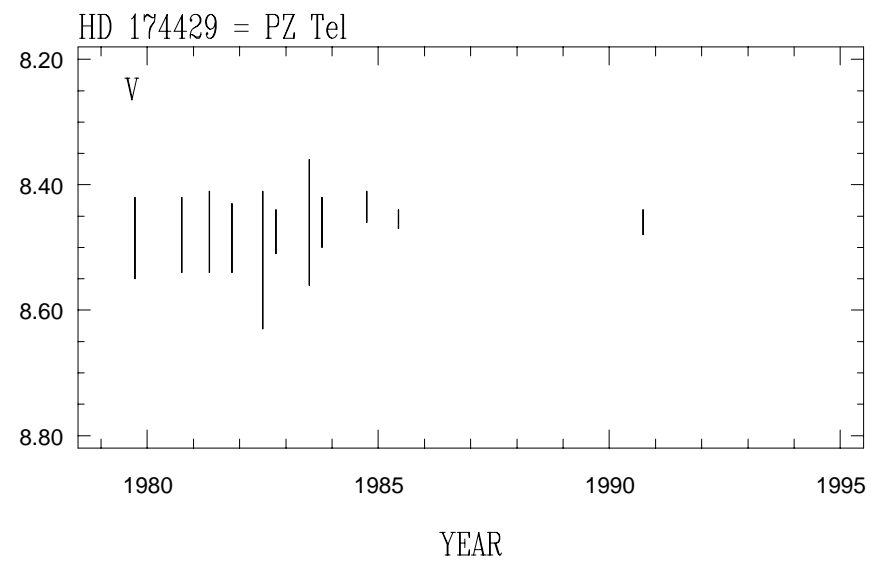

Fig. 15. $V$ light long-term variability of HD $217344=$ TZ PsA. The vertical bars indicate the peak-to-peak amplitude of the light curve

days (Herbig \& Moorhead 1965). The combined spectral type is given as dM2e and M0Ve by Joy \& Abt (1974) and Houk \& Smith-Moore (1988), respectively. FK Aqr has been observed by several authors showing the classical behavior of flare stars (see Cutispoto 1991 and references therein). More recently, evidence for X-ray flaring activity has been reported by Pollok et al. (1991) and the star has been detected at EUV wavelengths by the ROSAT (Pounds et al. 1993; Pye et al. 1995) and EUVE (Malina et al. 1994) satellites. The observations carried out over the interval 7-17 Sep. with the $0.5 \mathrm{~m}$ and over the interval 30 Sep. - 9 Oct. with the $1.0 \mathrm{~m}$ are shown in Fig. 13. Any light contribution from the optical companion GL 867 B $=$ FL Aqr, that lies 24.5 arsec apart, was avoided. Phases are computed by using the $4.252 \pm 0.044$-day period inferred from a Fourier analysis of the VRI data. We note that this photometric period is considerably shorter than the 4.39-day value computed by Byrne et al. (1987), a difference that is indicative of the presence of differential rotation. Moreover, the light curve in Fig. 13 is doublepeaked, while the one obtained less that 10 months before (Cutispoto 1995) was single-peaked. As the data in Cutispoto (1995) were folded by using the 4.39-day period, it is evident that strong modifications of the starspots' distribution, and in particular of the spot latitude, have occurred. The present $B-V, V-R$ and $V-I$ color indices are clearly correlated with the $V$-band modulation, while the more scattered $U-B$ color data seem to be anticorrelated. The colors are consistent with the combined M2 Ve spectral classification.

HD $217344=$ TZ PsA is an SB1 system with an orbital period of 1.643 days (Balona 1987) showing Ca II $\mathrm{H} \& \mathrm{~K}$ emission lines (Houk 1982). It was detected during a microwave survey of southern active stars (Slee et al. 1987) and during the Extended Medium Sensitivity Survey by the Einstein satellite (Gioia et al. 1990). Low Li abundance is reported by Favata et al. (1995) and optical variability was discovered by Lloyd Evans \& Koen (1987). The observations carried out using the $0.5 \mathrm{~m}$ over the interval 7-14 Sep. are shown in Fig. 14. Phases are reckoned from the 1.648-day photometric period computed by Lloyd Evans \& Koen (1987). The faint optical companion $(\Delta V \simeq 3.9, d \simeq 3.7$ arcsec) noted by Houk (1982) was always included into the diaphragm of our photometer. The low amplitude light curve appears rather complicated and weak color variations are present. A collection of the published photometry of HD 217344 is shown in Fig. 15. The few data available do not allow to draw any conclusion on the presence of a long-term variability of the degree of spottedness. Assuming the G5 V spectral classification reported by Houk (1982) for the primary component of HD 217344, the observed colors are very well matched by considering a G5 V + K7-M0 V system. It should be noted that, being HD 217344 a SB1 system, the inferred K7-M0 V component has to be considered as the combination of the optical companion plus the true physical companion. However, Favata et al. (1995) computed a $v \sin i$ of $72 \mathrm{~km} \mathrm{~s}^{-1}$, that leads to a minimum radius for the primary component of $2.34 R_{\odot}$ i.e. to a luminosity class IV star. A good match of the observed colors is then obtained by assuming a G5/6 IV primary component.

Acknowledgements. Stellar activity research at Catania Astrophysical Observatory is supported by the Italian Ministry for Scientific Research and Technology (MURST), the Italian National Council for Research (CNR Gruppo Nazionale di Astronomia) and the Sicilian Regional Government (Regione Sicilia) that are gratefully acknowledged. The authors thank the referee K.G. Strassmeier for his constructive comments and Ms. G. Santagati for revising the manuscript. This research has made use of the SIMBAD database, operated at CDS, Strasbourg, France. A special thank is also due to the ESO staff for the collaboration and technical support during the observations.

\section{References}

Balona L.A., 1987, S. Afr. Astron. Obs. Circ. 11, 1

Bidelman W.P., MacConnell D.J., 1973, AJ 78, 687

Bopp B.W., Africano J., Quigley R., 1986, AJ 92, 1409

Budding E., 1985, IAU Comm. 27, Inf. Bull. Var. Stars, No. 2779

Budding E., Zeilik M., 1995, Ap\&SS 232, 355

Bunton J.D., Large M.I., Slee O.B., et al., 1989, Proc. ASA 8, 127

Byrne P.B., Black E., Thè P.S., 1987, A\&A 186, 268

Byrne P.B., Agnew D.J., Cutispoto G., et al., 1992, in "Surface Inhomogeneities in Late-Type Stars", Byrne P.B. \& Mullan D.J. (eds.) Lecture Notes in Physics. Springer-Verlag, p. 255

Coates D.W., Halprin L., Sartori P., Thompson K., 1980, IAU Comm. 27 Inf. Bull. Var. Stars, No. 1849

Coates D.W., Halprin L., Sartori P., Thompson K,. 1983, MNRAS 202, 427

Collier A.C., 1982, Southern Stars 30, 177 
Collier Cameron A., 1987, S. Afr. Astron. Obs. Circ. 11, 57

Cutispoto G., 1991, A\&AS 89, 435

Cutispoto G., 1992, A\&AS 95, 397

Cutispoto G., 1995, A\&AS 111, 507

Cutispoto G., 1996, A\&AS (in press)

Cutispoto G., Rodonó M., 1992, in "The Solar Cycle", Harvey K.L. (ed.) ASP Conf. Ser. 27, p. 465

Cutispoto G., Pagano I., Rodonò M., 1992, A\&A 263, L3

Cutispoto G., Tagliaferri G., Pallavicini R., Pasquni L., Rodonò M., 1996, A\&AS 115, 41

Evans D.S., 1959, MNRAS 119, 526

Favata F., Barbera M., Micela G., Sciortino S., 1995, A\&A 295, 147

Gioia I.M., Maccacaro T., Schild R.E., et al., 1990, ApJS 72, 567

Golub L., 1983, in "Activity in Red-Dwarf Stars", IAU Coll. 71, Byrne P.B. and Rodonò M. (eds.). D. Reidel Publ. Comp., p. 83

Herbig G.H., Moorhead J.M., 1965, ApJ 141, 649

Houdebine E.R., Doyle J.G., 1994, A\&A 289, 185

Houk N., 1978, "Michigan Catalogue of two dimensional spectral types for the HD stars", Vol. 2, Department of Astronomy University of Michigan, Ann Arbor

Houk N., 1982, "Michigan Catalogue of two dimensional spectral types for the HD stars", Vol. 3, Department of Astronomy University of Michigan, Ann Arbor

Houk N., Smith-Moore M., 1988, "Michigan Catalogue of two dimensional spectral types for the HD stars", Vol. 4, Department of Astronomy University of Michigan, Ann Arbor

Innis J.L., Thompson K., Coates D.W., 1986, MNRAS 223, 183

Innis J.L., Coates D.W., Thompson K., 1988, MNRAS 233, 887

Innis J.L., Coates D.W., Thompson K., Lloyd Evans T., 1990, MNRAS 242, 306

Joy A.H., Abt H.A., 1974, ApJS 28, 1

Kürster M., Schmitt J.H.M.M., Cutispoto G., 1994, A\&A 289, 899

Landolt A.U., 1983, AJ 88, 439

Linsky J.L., Bornmann P.L., Carpenter K.G., et al., 1982, ApJ 260, 670

Lloyd Evans T., Koen M.C.J., 1987, S. Afr. Astron. Obs. Circ. 11,21
Malina R.F., Marshall H.L., Antia B., et al., 1994, AJ 107, 751

Martin E.L., Brandner W., 1995, A\&A 294, 744

Menzies J.W., Laing J.D., 1988, MNRAS 231, 1047

Menzies J.W., Cousins A.W.J., Banfiels R.M., Laing J.D., 1989, S. Afr. Astr. Obs. Circ. 13, 1

Pagano I., Rodonò M., Neff J.E., 1992, in "Surface Inhomeogeneities in Late-Type Stars. Byrne P.B. \& Mullan D.J. (eds.) Lecture Notes in Physics. Springer-Verlag, p. 315

Pagano I., Rodonò M., Cutispoto G., et al., 1993, in "Physics of Solar and Stellar Coronae", Linsky J.F. \& Serio S. (eds.). Kluwer Academic Publishers, p. 457

Pallavicini R., Cutispoto G., Randich S., Gratton R., 1993, A\&A 267, 145

Pallavicini R., Randich S., Giampapa M.S., 1992, A\&A 253, 185

Pasquini L., Cutispoto G., Gratton R., Mayor M., 1991, A\&A 248, 72

Pollock A.M.T., Tagliaferri G., Pallavicini R., 1991, A\&A 241, 451

Pounds K.A., Allan D.J., Barber C., et al., 1993, MNRAS 260, 77

Pye J.P., McGale P.A., Allan D.J., et al., 1995, MNRAS 274, 1165

Quin D.A., Doyle J.G., Butler C.J., Byrne P.B., Swank J.H., 1993, A\&A 272, 477

Randich S., Gratton R., Pallavicini R., 1993, A\&A 273, 194

Rodonò M., 1986, in "Highlights of Astronomy", Vol. 7, Swings J.-P. (ed.). Reidel Dordrecht, p. 429

Rodonò M., 1992a, in "Surface Inhomogeneities in Late-Type Stars" Byrne P.B. \& Mulland D.J. (eds.) Lect. Notes Phys. Springer-Verlag, p. 201

Rodonò M. 1992b, in "Evolutionary Processes in Interacting Binary Stars", Proc. 151st IAU Sym., Kondo Y. et al. (eds.). Kluwer Academic Publishers, p. 71

Rodonò M., Cutispoto G., 1992, A\&AS 95, 55

Rodonò M., Byrne P.B., Neff J.E., et al., 1987, A\&A 176, 267

Slee O.B., Nelson G.J., Steward R.T., et al., 1987, MNRAS 229,659

Strassmeier K.G., Hall D.S., Fekel F.C., Scheck M., 1993, A\&AS 100, 173

Torres C.A.O., Ferraz Mello S., Quast G.R., 1972, Astrophys. Lett. 11, 13 